\title{
UNIQUENESS OF ISOMETRIC IMMERSIONS WITH THE SAME MEAN CURVATURE
}

\author{
CHUNHE LI, PENGZI MIAO, AND ZHIZHANG WANG
}

\begin{abstract}
Motivated by the quasi-local mass problem in general relativity, we study the rigidity of isometric immersions with the same mean curvature into a warped product space. As a corollary of our main result, two star-shaped hypersurfaces in a spatial Schwarzschild or AdS-Schwarzschild manifold with nonzero mass differ only by a rotation if they are isometric and have the same mean curvature. We also prove similar results if the mean curvature condition is replaced by an $\sigma_{2}$-curvature condition.
\end{abstract}

\section{InTRODUCTION AND STATEMENT OF THE RESUlTS}

In the quasi-local mass problem in general relativity, a basic pair of geometric data $(g, H)$ associated to a 2 -sphere $\Sigma$, bounding some spacelike hypersurface $\Omega$ in a spacetime, consists of a Riemannian metric $g$ and a function $H$. Here $g$ denotes the induced metric on $\Sigma$ from $\Omega$ and $H$ is the mean curvature of $\Sigma$ in $\Omega$. For instance, the Brown-York quasi-local mass [6, 7] is given by

$$
\mathfrak{m}_{B Y}(\Sigma)=\frac{1}{8 \pi}\left(\int_{\Sigma} H_{0}-\int_{\Sigma} H\right),
$$

where the metric $g$ is assumed to have positive Gauss curvature and $H_{0}$ is the mean curvature of the unique, isometric embedding of $(\Sigma, g)$ into the 3-dimensional Euclidean space $\mathbb{R}^{3}$.

Existence and uniqueness of the isometric embedding of $(\Sigma, g)$ into $\mathbb{R}^{3}$, used in defining $\mathfrak{m}_{B Y}(\Sigma)$, was guaranteed by Nirenberg's solution to the Weyl embedding problem (cf. [29, 32, 33, 39]). When the target space $\mathbb{R}^{3}$ is replaced by the Minkowski spacetime $\mathbb{R}^{3,1}$, existence of isometric embeddings of $(\Sigma, g)$ into $\mathbb{R}^{3,1}$ was given by Wang and Yau [40, 41] in association with the definition of Wang-Yau quasi-local mass. If the target space is a general Riemannian 3-manifold $N$, estimates concerning the existence of isometric embeddings into $N$ have been studied extensively in the literature. We refer readers to the work in [9, 19, 24, 25, 30, 34, 35] and the references therein.

2010 Mathematics Subject Classification. Primary 53C45; Secondary 83C99.

Key words and phrases. Isometric embedding, mean curvature, quasi-local mass.

Research of the second author is partially supported by Simons Foundation Collaboration Grant for Mathematicians \#281105.

Research of the third author is partially supported by NSFC Grants No.11301087, No.11671090 and No.11771103. 
In contrast to the embedding into $\mathbb{R}^{3}$, counterexamples were constructed in [24] to illustrate the lack of rigidity for convex surfaces if the target Riemannian manifold is not a space form. Such non-uniqueness, however, does not diminish the role played by isometric embeddings in the study of relativistic problems. In [26], isometric embeddings of $(\Sigma, g)$ into a spatial Schwarzschild 3-manifold

$$
\left(N_{m}, d s_{m}^{2}\right)=\left([2 m, \infty) \times \mathbb{S}^{2}, \frac{1}{1-\frac{2 m}{r}} d r^{2}+r^{2} d \sigma\right)
$$

were used in deriving a localized Riemannian Penrose inequality [4, 22] that has a form of

$$
m+\frac{1}{8 \pi} \int_{\Sigma}\left(H_{m}-H\right) f \geq \sqrt{\frac{A}{16 \pi}} .
$$

Here $H_{m}$ is the mean curvature of an embedding of $(\Sigma, g)$ in $\left(N_{m}, d s_{m}^{2}\right), f$ is the static potential on $\left(N_{m}, d s^{2}\right)$ given by $f=\left(1-\frac{2 m}{r}\right)^{\frac{1}{2}}$, and $A$ represents the area of the horizon of black hole enclosed by $(\Sigma, g)$ in a physical manifold $\Omega$.

The Schwarzschild manifold $\left(N_{m}, d s_{m}^{2}\right)$ is an example of a static space $\left(N, d s^{2}\right)$, which by definition is a Riemannian 3-manifold on which there exists a function $f>0$, referred as a static potential, such that the corresponding static spacetime

$$
(\bar{N}, \bar{g})=\left(\mathbb{R}^{1} \times N,-f^{2} d t^{2}+d s^{2}\right)
$$

is Einstein. In [10], isometric embeddings of $(\Sigma, g)$ into such a static Einstein spacetime $(\bar{N}, \bar{g})$ were used as references in defining an analogue of the Wang-Yau mass. When the image of such an embedding lies in a constant $t$-slice of $(\bar{N}, \bar{g})$, i.e. in $\left(N, d s^{2}\right)$, the associated quasi-local energy in [10] becomes the integral

$$
\frac{1}{8 \pi} \int_{\Sigma}\left(H_{s}-H\right) f
$$

where $H_{s}$ is the mean curvature of the embedding of $(\Sigma, g)$ in $\left(N, d s^{2}\right)$.

Motivated by the non-uniqueness example of isometric embeddings in [24] and by the results in [10, 26] that make use of embeddings into static spaces, we ask the following question:

Question 1.1. In a static Riemannian 3-manifold $\left(N, d s^{2}\right)$, if two surfaces $\Sigma_{1}$ and $\Sigma_{2}$ are isometric and have the same mean curvature (under the surface isometry), do $\Sigma_{1}$ and $\Sigma_{2}$ differ only by a rigid motion of the ambient space?

We recall that the static condition on $\left(N, d s^{2}\right)$ can be formulated equivalently (cf. [16]) in Riemannian terms by

$$
(\tilde{\Delta} f) \tilde{g}-\tilde{\nabla}^{2} f+f \operatorname{Ric}(\tilde{g})=0
$$

where $\tilde{\Delta}, \tilde{\nabla}^{2}$ denote the Laplacian, the Hessian of $\tilde{g}=d s^{2}$, respectively, and $\operatorname{Ric}(\tilde{g})$ is the Ricci curvature of $\tilde{g}$. 
In this paper, we study Question 1.1 by considering embeddings of an $n$-sphere $\mathbb{S}^{n}$, $n \geq 2$, into an $(n+1)$-dimensional warped product space

$$
\left(N, d s^{2}\right)=\left(I \times \mathbb{S}^{n}, \frac{1}{f^{2}(r)} d r^{2}+r^{2} d \sigma\right)
$$

where $I \subset \mathbb{R}^{+}$is an interval, $f$ is a positive function on $I$, and $d \sigma$ denotes the standard metric of constant sectional curvature 1 on $\mathbb{S}^{n}$. In this case, a conformal Killing vector filed on $\left(N, d s^{2}\right)$ is $X=r f \frac{\partial}{\partial r}$. We define new radial coordinates $\rho$ and $u$ on $I$ by

$$
\rho=\frac{1}{2} X \cdot X=\frac{1}{2} r^{2} \text { and } u=\int \frac{d \rho}{f(\rho)},
$$

where "." denotes the metric $d s^{2}$. We also define

$$
\Phi=\frac{1}{2 \rho}\left(f f_{\rho}+\frac{1-f^{2}}{2 \rho}\right) .
$$

Direct calculation shows that $\left(N, d s^{2}\right)$ is static with $f$ satisfying (1.1) if and only if

$$
f_{u u}+(n-1) \Phi f=0 .
$$

Here $f_{u}, f_{\rho}, f_{u u}, f_{\rho \rho}$ denote the first, second derivatives of $f$ with respect to $u, \rho$, respectively.

Given an immersion $\iota: \mathbb{S}^{n} \rightarrow\left(N, d s^{2}\right)$, let $\nu$ be a chosen unit vector field normal to the hypersurface $M=\iota\left(\mathbb{S}^{n}\right)$, the support function of $M$ is defined by

$$
\varphi=X \cdot \nu
$$

With these notations, we can state the main result of this paper.

Theorem 1.1. Suppose $\left(N, d s^{2}\right)=\left(I \times \mathbb{S}^{n}, \frac{1}{f^{2}(r)} d r^{2}+r^{2} d \sigma\right)$ satisfies

$$
f_{u u}+(n-1) \Phi f \leq 0
$$

with either $\Phi \geq 0$ or $\Phi \leq 0$. Let $g$ be a Riemannian metric on $\mathbb{S}^{n}$. Suppose $\iota$ and $\tilde{\iota}$ are two isometric immersions of $\left(\mathbb{S}^{n}, g\right)$ into $\left(N, d s^{2}\right)$ such that $H=\tilde{H}$, where $H, \tilde{H}$ are the mean curvatures of the immersed surfaces $M=\iota\left(\mathbb{S}^{n}\right), \tilde{M}=\tilde{\iota}\left(\mathbb{S}^{n}\right)$, respectively. If $M$ and $\tilde{M}$ have positive support functions, then

$$
h_{i j}=\tilde{h}_{i j},
$$

where $h_{i j}, \tilde{h}_{i j}$ are the second fundamental forms of $M, \tilde{M}$, respectively.

Moreover, if $\Phi$ is strictly positive or negative, then $M$ and $\tilde{M}$ only differ by a rotation in $\left(N, d s^{2}\right)$.

Remark 1.1. The assumption that $\Phi$ is positive or negative agrees with the assumption (H4) or (H4') in Brendle's work on CMC surfaces in warped product spaces [5]. On the other hand, the assumption (1.7) appears to be opposite to the assumption (H3) or (H3') in [5]. 
Remark 1.2. When equality in (1.7) holds, i.e. when $\left(N, d s^{2}\right)$ is static, it is known that $f(r)$ is explicitly given by

$$
f=\left(1-2 m r^{1-n}+\kappa r^{2}\right)^{\frac{1}{2}}
$$

for some constants $m$ and $\kappa$. In this case, $\left(N, d s^{2}\right)$ includes the spatial Schwarzschild and AdS-Schwarzschild manifolds and $\Phi=(n+1) m r^{-n-3}$.

As a direct corollary of Theorem 1.1 and Remark 1.2, we have

Corollary 1.1. Let $\left(N, d s^{2}\right)$ be a spatial Schwarzschild or AdS-Schwarzschild manifold with nonzero mass. If $M$ and $\tilde{M}$ are two star-shaped hypersurfaces in $N$ such that $M$ and $\tilde{M}$ are isometric and have the same mean curvature, then $M$ and $\tilde{M}$ only differ by a rotation in $\left(N, d s^{2}\right)$.

Remark 1.3. The spatial Schwarzschild manifold $\left(N_{m}, d s_{m}^{2}\right)$ is an example of an asymptotically flat manifold (cf. [4, 22, 42]). If the ambient manifold $\left(N, d s^{2}\right)$ in Question 1.1 is asymptotically flat and static, we suspect its answer is positive under suitable conditions on the surface. This is tied to the uniqueness aspect of the static metric extension conjecture formulated for the Bartnik quasi-local mass [2].

When the target space is a space form, we note that the study of isometrically immersed surfaces with the same mean curvature has a longstanding history. A surface $S$ is called locally $H$-deformable if any point $x \in S$ has a neighborhood $U$ that admits a nontrivial 1-parameter family of isometric deformations which preserve the mean curvature. Results on locally $H$-deformable surfaces can be found in [8, 12 , 13, 23, 27, 36, 37, 38, 43] and references therein. Regarding global rigidity, Lawson and Tribuzy [28] proved that, for any compact oriented surface $\Sigma$ equipped with a Riemannian metric $g$ and given a non-constant function $H$ on $\Sigma$, there exist at most two geometrically distinct isometric immersions of $(\Sigma, g)$ into a space form, with the mean curvature function $H$.

The uniqueness of surfaces with prescribed extrinsic curvature alone is also a classic problem in differential geometry. For instance, the rigidity of constant mean curvature hypersurfaces is an example of the prescribed mean curvature problem. A theorem due to Alexandrov [1] asserts that any closed, embedded hypersurface in $\mathbb{R}^{n}$ with constant mean curvature is a round sphere. Montiel [31] proved a uniqueness theorem for starshaped hypersurfaces of constant mean curvature in certain rotationally symmetric manifolds. In [5], Brendle obtained a generalization of Alexandrov's theorem for a class of warped product manifolds. In general, if one poses some function on the exterior unit normal vector field of a convex hypersurface in the Euclidean space, the uniqueness is still open except for the 2-dimensional case (cf. [20]).

Prompted by Theorem 1.1, we also consider the rigidity question for isometrically immersed hypersurfaces with the same $\sigma_{2}$-curvature (see definition (5.1)). We have the following result. 
Theorem 1.2. Let $\left(N, d s^{2}\right)=\left(I \times \mathbb{S}^{n}, \frac{1}{f^{2}(r)} d r^{2}+r^{2} d \sigma\right)$ be a warped product space with $\Phi \Phi_{u}>0$. Let $g$ be a Riemannian metric on $\mathbb{S}^{n}$. Suppose $\left(\mathbb{S}^{n}, g\right)$ can be isometrically immersed into $\left(N, d s^{2}\right)$ as two hypersurfaces $M$ and $\tilde{M}$. If $M$ and $\tilde{M}$ have the same $\sigma_{2}$-curvature, then they differ only by a rotation in $\left(N, d s^{2}\right)$.

The remainder of this paper is organized as follows. In Section 2, we collect some basic formulae on immersed hypersurfaces in a warped product space. In Section 3, we prove the infinitesimal rigidity of isometric surfaces with the same mean curvature via integral identities. The method is then revised in Section 4 to derive the global rigidity, hence proving Theorem 1.1. In Section [5, we consider the analogue of Theorem 1.1 with the mean curvature condition replaced by the $\sigma_{2}$-curvature condition and prove Theorem 1.2. As an additional application of the method used in Sections 3 and 4 , we also give another proof of the infinitesimal rigidity and global rigidity of convex surfaces in space forms (see Theorem 5.3).

We recently have learned that Po-Ning Chen and Xiangwen Zhang [11] proved a rigidity result for surfaces in 3-dimensional spatial Schwarzschild manifold which is similar but different to that in Corollary 1.1. We want to thank the authors for the communication on their paper.

\section{Preliminaries on hypersurfaces in a WARPED Product SPACE}

Let $\Sigma=\mathbb{S}^{n}$ and let $g$ be a Riemannian metric on $\Sigma$. Suppose $\iota:(\Sigma, g) \rightarrow\left(N, d s^{2}\right)$ is an isometric immersion, where $\left(N, d s^{2}\right)$ is given in (1.2). Let $M=\iota(\Sigma)$ and let $D$ denote the Levi-Civita connection on $\left(N, d s^{2}\right)$. Let $\rho, u, \Phi, \nu$ and $\varphi$ be given in (1.3) - (1.6).

Suppose $\left\{e_{1}, e_{2}, \cdots, e_{n}\right\}$ is a local frame on $(\Sigma, g)$, which can be viewed as a frame on $M$ via $\iota$. Let $h_{i j}$ denote the second fundamental form of $M$, defined by

$$
D_{e_{i}} e_{j}=-h_{i j} \nu
$$

The following formulae can be easily checked (cf. [18, 24]):

$$
\begin{aligned}
\varphi^{2} & =2 \rho-\frac{|\nabla \rho|^{2}}{f^{2}}=2 \rho-|\nabla u|^{2}, \\
h_{i j} \varphi & =-\frac{\rho_{i, j}}{f}+\frac{f_{\rho}}{f^{2}} \rho_{i} \rho_{j}+f g_{i j}=-u_{i, j}+f g_{i j}, \\
\varphi_{i} & =\sum_{k} h_{i k} X \cdot e_{k}=\sum_{k} h_{i k} \frac{\rho_{k}}{f}=\sum_{k} h_{i k} u_{k} .
\end{aligned}
$$

Here a function with a lower index $i$ denotes its derivative along $e_{i}$ and ", "denotes the covariant differentiation on $M$ or equivalently on $(\Sigma, g)$.

Given vector fields $Y, Z, W$ on $N$, let the curvature tensor on $\left(N, d s^{2}\right)$ be given by

$$
\tilde{R}(Y, Z) W=D_{Y} D_{Z} W-D_{Z} D_{Y} W-D_{[Y, Z]} W
$$


Direct calculation (see (2.7) in [24]) shows

$$
\begin{aligned}
(-1) \sum_{i<j} \tilde{R}\left(e_{i}, e_{j}\right) e_{j} \cdot e_{i} & =(n-1)\left[f f_{\rho}+\frac{n-2}{4} \frac{f^{2}-1}{\rho}-\varphi^{2} \frac{2 \rho f f_{\rho}+1-f^{2}}{4 \rho^{2}}\right] \\
& =(n-1)\left(f_{u}+\frac{n-2}{4} \frac{f^{2}-1}{\rho}-\varphi^{2} \Phi\right) \\
& =(n-1)\left[\frac{n}{2} f_{u}-(n-2) \rho \Phi-\varphi^{2} \Phi\right] .
\end{aligned}
$$

Let $R$ be the scalar curvature of $(\Sigma, g)$. By the Gauss equations, we have

$$
\sigma_{2}(h)=\frac{R}{2}+(n-1)\left[\frac{n}{2} f_{u}-(n-2) \rho \Phi-\varphi^{2} \Phi\right] .
$$

Here $\sigma_{2}(h)=\sum_{i<j} \kappa_{i} \kappa_{j}$, where $\left\{\kappa_{i}\right\}_{i=1}^{n}$ are the principal curvature of $M$. The Codazzi equations are given by

$$
h_{i j, k}-h_{i k, j}=\tilde{R}\left(e_{j}, e_{k}\right) e_{i} \cdot \nu
$$

which implies

$$
\sum_{i} h_{i j, i}-H_{j}=\tilde{R i c_{j \nu}}
$$

Here $\tilde{\operatorname{Ric}}=\operatorname{Ric}(\tilde{g})$ denotes the Ricci curvature of $\left(N, d s^{2}\right)$. The following formula can be checked:

$$
\tilde{R i} c_{j \nu}=-(n-1) \varphi \Phi u_{j} .
$$

(For instance, (2.8) follows from (2.3) in [24].) Thus, (2.7) becomes

$$
\sum_{i} h_{i j, i}-H_{j}=-(n-1) \varphi \Phi u_{j}
$$

The geometric meaning of $\Phi$ is as follows:

$$
\tilde{\operatorname{Ric}}\left(E_{1}, E_{1}\right)-\tilde{\operatorname{Ric}}(V, V)=-(n-1) 2 \rho \Phi,
$$

where $E_{1}=f \partial_{r}$ is the unit normal to $S_{r}=\{r\} \times \mathbb{S}^{n}$ and $V$ denotes any unit vector tangent to $S_{r}$. In relation to equation (1.1), we also note

$$
\left[(\tilde{\Delta} f) \tilde{g}-\tilde{\nabla}^{2} f+f \tilde{R i c}\right](V, V)=2 \rho\left[f_{u u}+(n-1) \Phi f\right] .
$$

By (1.4), $\Phi$ can be rewritten as

$$
\Phi=\frac{1}{4}\left(\frac{f^{2}-1}{\rho}\right)_{\rho} .
$$

From this, it is easily seen

$$
\left(f f_{\rho}\right)_{\rho}-4 \Phi-2 \rho \Phi_{\rho}=0
$$

or equivalently

$$
f_{u u}-4 \Phi f-2 \rho \Phi_{u}=0 .
$$




\section{INFINITESIMAL RIGIDITY FOR SURFACES WITH FIXED MEAN CURVATURE}

In this section, we prove the infinitesimal rigidity for isometric surfaces with the same mean curvature. Suppose $\left\{\iota_{t}\right\}$ is a 1-parameter family of isometric immersions of $(\Sigma, g)$ in $\left(N, d s^{2}\right)$ which have the same mean curvature function $H$. Let $M_{t}=\iota_{t}(\Sigma)$ and $M=M_{0}$. Let an upper dot denote derivative with respect to $t$ at $t=0$. Then

$$
\dot{H}=\left.\frac{d H}{d t}\right|_{t=0}=0 ; \quad \dot{g}_{i j}=\left.\frac{d g_{i j}}{d t}\right|_{t=0}=0 .
$$

The infinitesimal rigidity means that we want to show $\dot{h}_{i j}=0$.

Let $\left\{e_{i}\right\}_{1 \leq i \leq n}$ be a local orthonormal frame on $(\Sigma, g)$. By (2.1) and (2.2), we have

$$
\begin{gathered}
\varphi \dot{\varphi}=f \dot{u}-\nabla u \cdot \nabla \dot{u}, \\
\dot{h}_{i j} \varphi+h_{i j} \dot{\varphi}=-\dot{u}_{i, j}+f_{u} \dot{u} \delta_{i j} .
\end{gathered}
$$

The linearization of (2.5) and (2.9) implies

$$
\begin{aligned}
& \sum_{i, j} h_{i j} \dot{h}_{i j}=H \dot{H}-\dot{\sigma}_{2}(h) \\
= & -(n-1)\left(\frac{n}{2} f_{u u} \dot{u}-(n-2) f \Phi \dot{u}-(n-2) \rho \Phi_{u} \dot{u}-2 \varphi \dot{\varphi} \Phi-\varphi^{2} \Phi_{u} \dot{u}\right),
\end{aligned}
$$

$$
\sum_{i} \dot{h}_{i j, i}=-(n-1)\left(\dot{u}_{j} \varphi \Phi+u_{j} \dot{\varphi} \Phi+u_{j} \varphi \Phi_{u} \dot{u}\right)
$$

where we have used $\dot{H}=0$.

Let $w$ be an auxiliary function defining on $I$, which will be determined later. Viewing $w$ as a function on $N$ and pulling it back to $\Sigma$, we have by (3.3),

$$
\dot{h}_{i j} \varphi w=-\dot{u}_{i, j} w+f_{u} \dot{u} w \delta_{i j}-h_{i j} w \dot{\varphi}
$$


Integrating on $(\Sigma, g)$ and using $\dot{H}=0$, we have

$$
\begin{aligned}
\int_{\Sigma} \sum_{i j} \dot{h}_{i j}^{2} \varphi w= & -\sum_{i, j} \int_{\Sigma} \dot{u}_{i, j} w \dot{h}_{i j}+\int_{\Sigma} \dot{u} w \dot{H}-\sum_{i, j} \int_{\Sigma} h_{i j} \dot{h}_{i j} w \dot{\varphi} \\
= & \sum_{i, j} \int_{\Sigma} \dot{u}_{i} w_{j} \dot{h}_{i j}+\sum_{i, j} \int_{\Sigma} \dot{u}_{i} w \dot{h}_{j i, j}-\sum_{i, j} \int_{\Sigma} h_{i j} \dot{h}_{i j} w \dot{\varphi} \\
= & -\sum_{i, j} \int_{\Sigma} \dot{u} w_{i, j} \dot{h}_{i j}-\sum_{i, j} \int_{\Sigma} \dot{u} w_{j} \dot{h}_{i j, i}+\sum_{i, j} \int_{\Sigma} \dot{u}_{i} w \dot{h}_{j i, j}-\sum_{i, j} \int_{\Sigma} h_{i j} \dot{h}_{i j} w \dot{\varphi} \\
= & -\sum_{i, j} \int_{\Sigma} \dot{u} w_{u u} u_{i} u_{j} \dot{h}_{i j}-\sum_{i, j} \int_{\Sigma} \dot{u} w_{j} \dot{h}_{i j, i}+\sum_{i, j} \int_{\Sigma} \dot{u}_{i} w \dot{h}_{j i, j}-\sum_{i, j} \int_{\Sigma} h_{i j} \dot{h}_{i j} w \dot{\varphi} \\
& +\sum_{i, j} \int_{\Sigma} w_{u} \dot{u}\left(-u_{i, j}+f \delta_{i j}\right) \dot{h}_{i j}-\int_{\Sigma} w_{u} \dot{u} f \dot{H} \\
= & -\sum_{i, j} \int_{\Sigma} \dot{u} w_{u u} u_{i} u_{j} \dot{h}_{i j}+\sum_{i, j} \int_{\Sigma}\left(\dot{u}_{i} w-\dot{u} w_{i}\right) \dot{h}_{j i, j}-\sum_{i, j} \int_{\Sigma} h_{i j} \dot{h}_{i j}\left(w \dot{\varphi}-w_{u} \dot{u} \varphi\right) .
\end{aligned}
$$

Applying (3.4) and (3.5), we then have

$$
\begin{aligned}
& \int_{\Sigma} \sum_{i j} \dot{h}_{i j}^{2} \varphi w \\
= & -\sum_{i, j} \int_{\Sigma} w_{u u} \dot{u} u_{i} u_{j} \dot{h}_{i j}-(n-1) \sum_{i} \int_{\Sigma}\left(\dot{u}_{i} w-\dot{u} w_{u} u_{i}\right)\left(\dot{u}_{i} \varphi \Phi+u_{i} \dot{\varphi} \Phi+u_{i} \varphi \Phi_{u} \dot{u}\right) \\
& +(n-1) \int_{\Sigma}\left(w \dot{\varphi}-w_{u} \dot{u} \varphi\right)\left(\frac{n}{2} f_{u u} \dot{u}-(n-2)\left(f \Phi+\rho \Phi_{u}\right) \dot{u}-2 \varphi \dot{\varphi} \Phi-\varphi^{2} \Phi_{u} \dot{u}\right) \\
= & -\sum_{i, j} \int_{\Sigma} w_{u u} \dot{u} u_{i} u_{j} \dot{h}_{i j}-(n-1) \int_{\Sigma}\left(|\nabla \dot{u}|^{2}+2 \dot{\varphi}^{2}\right) w \varphi \Phi \\
& +(n-1) \int_{\Sigma} \dot{u} \dot{\varphi}\left(|\nabla u|^{2} w_{u} \Phi+\frac{n}{2} w f_{u u}-(n-2)\left(f \Phi+\rho \Phi_{u}\right) w+2 \varphi^{2} w_{u} \Phi-w \varphi^{2} \Phi_{u}\right) \\
& -(n-1) \int_{\Sigma} \dot{u}^{2}\left(\frac{n}{2} w_{u} \varphi f_{u u}-(n-2)\left(f \Phi+\rho \Phi_{u}\right) w_{u} \varphi-|\nabla u|^{2} w_{u} \varphi \Phi_{u}-w_{u} \varphi^{3} \Phi_{u}\right) \\
& -(n-1) \int_{\Sigma} \nabla u \cdot \nabla \dot{u}\left(-\dot{u} w_{u} \varphi \Phi+\dot{\varphi} \Phi w+\dot{u} w \varphi \Phi_{u}\right) .
\end{aligned}
$$

To proceed, we note that

$$
\begin{aligned}
& \int_{\Sigma}|\nabla \dot{u}|^{2} w \varphi \Phi=\int_{\Sigma} w \varphi \Phi \nabla \dot{u} \cdot \nabla \dot{u} \\
= & -\int_{\Sigma} \dot{u}(\Delta \dot{u}) w \varphi \Phi-\int_{\Sigma} \dot{u} w \Phi \nabla \dot{u} \cdot \nabla \varphi-\int_{\Sigma} \dot{u} \varphi\left(w_{u} \Phi+w \Phi_{u}\right) \nabla \dot{u} \cdot \nabla u,
\end{aligned}
$$


and

$$
\begin{aligned}
& \int_{\Sigma} \dot{\varphi}^{2} \varphi w \Phi=\int_{\Sigma}(f \dot{u}-\nabla u \cdot \nabla \dot{u}) \dot{\varphi} w \Phi \\
= & \int_{\Sigma} \dot{u} \dot{\varphi} f w \Phi+\int_{\Sigma} \dot{u} \dot{\varphi} w \Phi \Delta u+\int_{\Sigma} \dot{u} w \Phi \nabla u \cdot \nabla \dot{\varphi}+\int_{\Sigma} \dot{u} \dot{\varphi}\left(w_{u} \Phi+w \Phi_{u}\right)|\nabla u|^{2} .
\end{aligned}
$$

Moreover, the linearization of (2.3) gives

$$
\dot{\varphi}_{i}=\sum_{k} \dot{h}_{i k} u_{k}+\sum_{k} h_{i k} \dot{u}_{k}
$$

Thus, we have

$$
\nabla u \cdot \nabla \dot{\varphi}-\nabla \dot{u} \cdot \nabla \varphi=\sum_{i, j} \dot{h}_{i j} u_{i} u_{j}
$$

It follows from (3.8) - (3.11) that

$$
\begin{aligned}
& \int_{\Sigma}\left(|\nabla \dot{u}|^{2}+\dot{\varphi}^{2}\right) w \varphi \Phi \\
= & \int_{\Sigma} w \Phi \dot{u} \dot{h}_{i j} u_{i} u_{j}+\int_{\Sigma} \dot{u} w \Phi(\dot{\varphi} \Delta u-\varphi \Delta \dot{u})-\int_{\Sigma} \dot{u} \varphi\left(w_{u} \Phi+w \Phi_{u}\right) \nabla u \cdot \nabla \dot{u} \\
& +\int_{\Sigma} \dot{u} \dot{\varphi}\left(f w \Phi+\left(w_{u} \Phi+w \Phi_{u}\right)|\nabla u|^{2}\right) .
\end{aligned}
$$

Using (2.2) and (3.3), we have

$$
\dot{\varphi} \Delta u-\varphi \Delta \dot{u}=\dot{\varphi}(n f-H \varphi)-\varphi\left(n f_{u} \dot{u}-H \dot{\varphi}\right)=n\left(f \dot{\varphi}-\varphi f_{u} \dot{u}\right),
$$

where $\dot{H}=0$ has been used. Thus (3.12) becomes

$$
\begin{aligned}
& \int_{\Sigma}\left(|\nabla \dot{u}|^{2}+\dot{\varphi}^{2}\right) w \varphi \Phi \\
= & \int_{\Sigma} w \Phi \dot{u} \dot{h}_{i j} u_{i} u_{j}-n \int_{\Sigma} \dot{u}^{2} w f_{u} \Phi \varphi-\int_{\Sigma} \dot{u} \varphi\left(w_{u} \Phi+w \Phi_{u}\right) \nabla u \cdot \nabla \dot{u} \\
& +\int_{\Sigma} \dot{u} \dot{\varphi}\left((n+1) f w \Phi+\left(w_{u} \Phi+w \Phi_{u}\right)|\nabla u|^{2}\right) .
\end{aligned}
$$


Combing (3.7) with (3.13), we obtain

$$
\begin{aligned}
& \int_{\Sigma} \sum_{i j} \dot{h}_{i j}^{2} \varphi w \\
= & -\sum_{i, j} \int_{\Sigma}\left(w_{u u}+(n-1) \Phi w\right) \dot{u} u_{i} u_{j} \dot{h}_{i j} \\
& -(n-1) \int_{\Sigma} \nabla u \cdot \nabla \dot{u}\left(-2 \dot{u} w_{u} \varphi \Phi+\dot{\varphi} \Phi w\right)-(n-1) \int_{\Sigma} \dot{\varphi}^{2} w \varphi \Phi \\
& -(n-1) \int_{\Sigma} \dot{u}^{2}\left(\frac{n}{2} w_{u} \varphi f_{u u}-(n-2)\left(f \Phi+\rho \Phi_{u}\right) w_{u} \varphi\right. \\
& -(n-1) \int_{\Sigma} \dot{u} \dot{\varphi}\left(-|\nabla u|^{2} w w_{u} \varphi \Phi_{u}-w_{u} \varphi^{3} \Phi_{u}-n w f_{u} \varphi \Phi\right) \\
& +2 f_{u u}-(n-2)\left(f \Phi+\rho \Phi_{u}\right) w
\end{aligned}
$$

Applying (3.2) and (2.1) to replace the terms $\nabla u \cdot \nabla \dot{u}$ and $|\nabla u|^{2}$ in (3.14), we have

$$
\begin{aligned}
& \int_{\Sigma} \sum_{i j} \dot{h}_{i j}^{2} \varphi w \\
= & -\sum_{i, j} \int_{\Sigma}\left(w_{u u}+(n-1) \Phi w\right) \dot{u} u_{i} u_{j} \dot{h}_{i j} \\
& +\frac{n(n-1)}{2} \int_{\Sigma} \dot{u} \dot{\varphi}\left(-2 \rho w \Phi_{u}+w f_{u u}-4 f w \Phi\right) \\
& -\frac{n(n-1)}{2} \int_{\Sigma} \dot{u}^{2}\left(w_{u} \varphi f_{u u}-2 \rho w_{u} \varphi \Phi_{u}-2 w f_{u} \varphi \Phi-2 f w_{u} \varphi \Phi\right) \\
= & -\sum_{i, j} \int_{\Sigma}\left(w_{u u}+(n-1) \Phi w\right) \dot{u} u_{i} u_{j} \dot{h}_{i j}-n(n-1) \int_{\Sigma} \dot{u}^{2} \varphi \Phi\left(w_{u} f-w f_{u}\right),
\end{aligned}
$$

where (2.12) has been used in the second equality. Thus, we obtain

$$
\begin{aligned}
0= & \int_{\Sigma} \sum_{i j} \dot{h}_{i j}^{2} \varphi w+\sum_{i, j} \int_{\Sigma}\left(w_{u u}+(n-1) \Phi w\right) \dot{u} u_{i} u_{j} \dot{h}_{i j} \\
& +n(n-1) \int_{\Sigma} \dot{u}^{2} \varphi \Phi\left(w_{u} f-w f_{u}\right) .
\end{aligned}
$$

If $\left(N, d s^{2}\right)$ is static with $f$ satisfying (1.1), we can choose $w=f$. In view of (1.5) and (3.16), we then conclude $\dot{h}_{i j}=0$ provided the support function $\varphi$ is positive.

Next suppose condition (1.7) holds. Let $\left(r_{0}, r_{1}\right) \subset I$ be an interval so that the coordinate $r$ varies between $r_{0}$ and $r_{1}$ on the surface $M$. We first consider the case 
$\Phi \geq 0$. In this case, let $w$ be a solution to the linear second order ordinary differential equation

$$
w_{u u}+(n-1) \Phi w=0
$$

with the initial conditions $w\left(r_{0}\right)>0, w_{u}\left(r_{0}\right)>0$ and

$$
w_{u}\left(r_{0}\right) f\left(r_{0}\right)-w\left(r_{0}\right) f_{u}\left(r_{0}\right) \geq 0 \text {. }
$$

(Note that such a $w$ always exists.) Then we have

$$
\left(\frac{w}{f}\right)_{u u}+2 \frac{f_{u}}{f}\left(\frac{w}{f}\right)_{u}+\frac{f_{u u}+(n-1) \Phi f}{f} \frac{w}{f}=0,
$$

where

$$
\frac{f_{u u}+(n-1) \Phi f}{f} \leq 0
$$

by (1.7). We claim that $w / f$ is always positive when $r$ ranges in $\left[r_{0}, r_{1}\right]$. To see this, suppose $r_{*} \in\left(r_{0}, r_{1}\right]$ is the first zero of $w / f$ from $r_{0}$. As $w_{u}\left(r_{0}\right)>0, \max _{r \in\left[r_{0}, r_{*}\right]} w / f$ must occur in $\left(r_{0}, r_{*}\right)$, which contradicts the strong maximum principle. Therefore, $w>0$ for $r \in\left(r_{0}, r_{1}\right)$. Consequently,

$$
\left(w_{u} f-w f_{u}\right)_{u}=w_{u u} f-w f_{u u}=-w\left(f_{u u}+(n-1) \Phi f\right) \geq 0,
$$

by (1.7). Hence, by (3.18),$w_{u} f-w f_{u} \geq 0$. This allows us to conclude $\dot{h}_{i j}=0$ from (3.16) under the assumption $\varphi>0$. If $\Phi>0$ on $N$, by choosing the inequality in (3.18) to be strict, we have $w_{u} f-w f_{u}>0$. In this case, it also follows from (3.16) that $\dot{u}=0$.

If $\Phi \leq 0$, similar arguments apply if we choose $w$ so that $w\left(r_{1}\right)>0, w_{u}\left(r_{1}\right)<0$ and

$$
w_{u}\left(r_{1}\right) f\left(r_{1}\right)-w\left(r_{1}\right) f_{u}\left(r_{1}\right) \leq 0 .
$$

When $\Phi<0$, we choose the inequality in (3.21) to be strict.

The above discussion has proved the following theorem.

Theorem 3.1. Suppose $\left(N, d s^{2}\right)=\left(I \times \mathbb{S}^{n}, \frac{1}{f^{2}(r)} d r^{2}+r^{2} d \sigma\right)$ satisfies (1.7), with either $\Phi \geq 0$ or $\Phi \leq 0$. Let $g$ be a Riemannian metric on $\Sigma=\mathbb{S}^{n}$. If $(\Sigma, g)$ can be isometrically immersed into $\left(N, d s^{2}\right)$ as a hypersurface $M$ with positive support function, then the infinitesimal rigidity of $M$ with fixed mean curvature holds. More precisely, this means that if $(\Sigma, g)$ admits a family of isometric immersion $\left\{\iota_{t}\right\}$ such that $M_{t}=\iota_{t}(\Sigma)$ has the same mean curvature and $M_{0}=M$, then the linearization of the second fundamental form of $M_{t}$ at $M=M_{0}$ is trivial. Moreover, if $\Phi>0$ or $\Phi<0$, the linearization of the function $u$ at $M=M_{0}$ is also trivial. 


\section{Global Rigidity for surfaces With FiXed mean CuRvature}

In this section, we will modify the proof in Section 3 to prove the global rigidity, i.e. Theorem 1.1. Suppose $\iota, \tilde{\iota}$ are two isometric immersions of $(\Sigma, g)$ in $\left(N, d s^{2}\right)$. Let $M=\iota(\Sigma)$ and $\tilde{M}=\tilde{\iota}(\Sigma)$. We denote the restriction of $X, \rho, u$ (which is defined in the ambient space $N$ ) to $\tilde{M}$ by $\tilde{X}, \tilde{\rho}, \tilde{u}$, respectively. By abuse of notations, we also use $X, \rho, u$ to denote their restriction to $M$. Let $\varphi, h$ and $\tilde{\varphi}, \tilde{h}$ be the support function, the second fundamental form of $M$ and $\tilde{M}$, respectively. Viewing $h$ and $\tilde{h}$ as tensors on $\Sigma$, we define

$$
v=\tilde{h}-h \text {. }
$$

Now suppose $M$ and $\tilde{M}$ have the same mean curvature. Let $\left\{e_{1}, e_{2}, \cdots, e_{n}\right\}$ be a local orthonormal frame on $(\Sigma, g)$, we have

$$
\sum_{i} v_{i i}=0
$$

By the Gauss equations of $M$ and $\tilde{M}$, the corresponding equations of (2.5) are

$$
\begin{aligned}
\sigma_{2}(\tilde{h}) & =\frac{\tilde{R}}{2}+(n-1)\left(\frac{n}{2} f_{u}(\tilde{u})-(n-2) \tilde{\rho} \Phi(\tilde{u})-\tilde{\varphi}^{2} \Phi(\tilde{u})\right), \\
\sigma_{2}(h) & =\frac{R}{2}+(n-1)\left(\frac{n}{2} f_{u}(u)-(n-2) \rho \Phi(u)-\varphi^{2} \Phi(u)\right) .
\end{aligned}
$$

Since $\tilde{R}=R$, the difference of the above two equations gives

$$
\begin{aligned}
& \sum_{i<j}\left(h_{i i} v_{j j}+h_{j j} v_{i i}-2 h_{i j} v_{i j}+v_{i i} v_{j j}-v_{i j}^{2}\right) \\
= & \frac{n(n-1)}{2}\left(f_{u}(\tilde{u})-f_{u}(u)\right)-(n-2)(n-1)(\tilde{\rho} \Phi(\tilde{u})-\rho \Phi(u)) \\
& -(n-1)\left(\tilde{\varphi}^{2} \Phi(\tilde{u})-\varphi^{2} \Phi(u)\right) .
\end{aligned}
$$

Using (4.1), we can rewrite the above as

$$
\begin{aligned}
-\sum_{i, j} h_{i j} v_{i j}= & \frac{(n-1) n}{2}\left(f_{u}(\tilde{u})-f_{u}(u)\right)-(n-2)(n-1)(\tilde{\rho} \Phi(\tilde{u})-\rho \Phi(u)) \\
& -(n-1)\left(\tilde{\varphi}^{2} \Phi(\tilde{u})-\varphi^{2} \Phi(u)\right)+\frac{1}{2} \sum_{i, j} v_{i j}^{2} \\
-\sum_{i, j} \tilde{h}_{i j} v_{i j}= & \frac{(n-1) n}{2}\left(f_{u}(\tilde{u})-f_{u}(u)\right)-(n-2)(n-1)(\tilde{\rho} \Phi(\tilde{u})-\rho \Phi(u)) \\
& -(n-1)\left(\tilde{\varphi}^{2} \Phi(\tilde{u})-\varphi^{2} \Phi(u)\right)-\frac{1}{2} \sum_{i, j} v_{i j}^{2} .
\end{aligned}
$$


By the Codazzi equations of $M$ and $\tilde{M}$, the corresponding equations of (2.9) are

$$
\begin{aligned}
& \sum_{i} h_{i j, i}-H_{j}=-(n-1) \varphi \Phi(u) u_{j}, \\
& \sum_{i} \tilde{h}_{i j, i}-\tilde{H}_{j}=-(n-1) \tilde{\varphi} \Phi(\tilde{u}) \tilde{u}_{j}
\end{aligned}
$$

which, combined with $H=\tilde{H}$, imply

$$
\sum_{i} v_{i j, i}=-(n-1)\left(\tilde{\varphi} \Phi(\tilde{u}) \tilde{u}_{j}-\varphi \Phi(u) u_{j}\right)
$$

We still let $w$ be some weighted single-variable function on $I$ which is to be chosen later. We calculate the following integral

$$
\begin{aligned}
& -\int_{\Sigma} \sum_{i, j}\left(w(\tilde{u}) u_{i}-w(u) \tilde{u}_{i}\right)_{j} v_{j i} \\
= & \int_{\Sigma} \sum_{i, j}\left(w(\tilde{u}) u_{i}-w(u) \tilde{u}_{i}\right) v_{j i, j} \\
= & (n-1) \int_{\Sigma} w(u) \tilde{\varphi} \Phi(\tilde{u})|\nabla \tilde{u}|^{2}+(n-1) \int_{\Sigma} w(\tilde{u}) \varphi \Phi(u)|\nabla u|^{2} \\
& -(n-1) \int_{\Sigma}(w(u) \varphi \Phi(u)+w(\tilde{u}) \tilde{\varphi} \Phi(\tilde{u})) \nabla u \cdot \nabla \tilde{u} .
\end{aligned}
$$

Here, in the second equality, (4.5) has been used.

On the other hand, we have

$$
\left(w(\tilde{u}) u_{i}-w(u) \tilde{u}_{i}\right)_{j}=w_{u}(\tilde{u}) \tilde{u}_{j} u_{i}-w_{u}(u) \tilde{u}_{i} u_{j}+w(\tilde{u}) u_{i j}-w(u) \tilde{u}_{i j}
$$

where, by (2.1),

$$
u_{i j}=f(u) \delta_{i j}-h_{i j} \varphi, \quad \tilde{u}_{i j}=f(\tilde{u}) \delta_{i j}-\tilde{h}_{i j} \tilde{\varphi} .
$$


Therefore,

$$
\begin{aligned}
& -\sum_{i, j}\left(w(\tilde{u}) u_{i}-w(u) \tilde{u}_{i}\right)_{j} v_{j i} \\
= & -\sum_{i, j}\left(w_{u}(\tilde{u}) \tilde{u}_{j} u_{i}-w_{u}(u) \tilde{u}_{i} u_{j}\right) v_{i j} \\
& +w(u) \sum_{i, j}\left(f(\tilde{u}) \delta_{i j}-\tilde{h}_{i j} \tilde{\varphi}\right) v_{i j}-w(\tilde{u}) \sum_{i, j}\left(f(u) \delta_{i j}-h_{i j} \varphi\right) v_{i j} \\
= & \left(w_{u}(u)-w_{u}(\tilde{u})\right) \sum_{i, j} \tilde{u}_{i} u_{j} v_{i j}+w(\tilde{u}) \varphi \sum_{i, j} h_{i j} v_{i j}-w(u) \tilde{\varphi} \sum_{i, j} \tilde{h}_{i j} v_{i j} \\
= & -\int_{u}^{\tilde{u}} w_{s s}(s) d s \sum_{i, j} u_{i} \tilde{u}_{j} v_{i j} \\
& +w(u) \tilde{\varphi}\left(\frac{(n-1) n}{2}\left(f_{u}(\tilde{u})-f_{u}(u)\right)-(n-2)(n-1)(\tilde{\rho} \Phi(\tilde{u})-\rho \Phi(u))\right. \\
& \left.+(n-1)\left(\varphi^{2} \Phi(u)-\tilde{\varphi}^{2} \Phi(\tilde{u})\right)-\frac{1}{2} \sum_{i, j} v_{i j}^{2}\right) \\
& -w(\tilde{u}) \varphi\left(\frac{(n-1) n}{2}\left(f_{u}(\tilde{u})-f_{u}(u)\right)-(n-2)(n-1)(\tilde{\rho} \Phi(\tilde{u})-\rho \Phi(u))\right. \\
& \left.+(n-1)\left(\varphi^{2} \Phi(u)-\tilde{\varphi}^{2} \Phi(\tilde{u})\right)+\frac{1}{2} \sum_{i, j} v_{i j}^{2}\right),
\end{aligned}
$$

where we have used (4.1), (4.4) in the second, the third equality, respectively. By (4.6) and (4.7), we thus have

$$
\begin{aligned}
0= & (n-1) \int_{\Sigma}\left[w(u) \tilde{\varphi} \Phi(\tilde{u})\left(|\nabla \tilde{u}|^{2}+\tilde{\varphi}^{2}\right)+w(\tilde{u}) \varphi \Phi(u)\left(|\nabla u|^{2}+\varphi^{2}\right)\right. \\
& -(w(u) \varphi \Phi(u)+w(\tilde{u}) \tilde{\varphi} \Phi(\tilde{u}))(\nabla u \cdot \nabla \tilde{u}+\varphi \tilde{\varphi})] \\
& +\int_{\Sigma} \sum_{i, j} u_{i} \tilde{u}_{j} v_{i j} \int_{u}^{\tilde{u}} w_{s s}(s) d s-\frac{(n-1) n}{2} \int_{\Sigma} w(u) \tilde{\varphi} \int_{u}^{\tilde{u}} f_{s s}(s) d s \\
& +\frac{(n-1) n}{2} \int_{\Sigma} w(\tilde{u}) \varphi \int_{u}^{\tilde{u}} f_{s s}(s) d s+\frac{1}{2} \int_{\Sigma}(w(u) \tilde{\varphi}+w(\tilde{u}) \varphi) \sum_{i, j} v_{i j}^{2} \\
& +(n-2)(n-1) \int_{\Sigma}(w(u) \tilde{\varphi}-w(\tilde{u}) \varphi) \int_{u}^{\tilde{u}}(\rho \Phi(s))_{s} d s .
\end{aligned}
$$


To handle the first integral on the right side of (4.8), we note that, by (2.1),

$$
\begin{aligned}
& w(u) \tilde{\varphi} \Phi(\tilde{u}) 2 \tilde{\rho}-w(u) \tilde{\varphi} \Phi(u) 2 \rho+w(\tilde{u}) \varphi \Phi(u) 2 \rho \\
& -w(\tilde{u}) \varphi \Phi(\tilde{u}) 2 \tilde{\rho}+w(u) \tilde{\varphi} \Phi(u) 2 \rho+w(\tilde{u}) \varphi \Phi(\tilde{u}) 2 \tilde{\rho} \\
& -(w(u) \varphi \Phi(u)+w(\tilde{u}) \tilde{\varphi} \Phi(\tilde{u}))(\nabla u \cdot \nabla \tilde{u}+\varphi \tilde{\varphi}) \\
= & w(u) \tilde{\varphi} \int_{u}^{\tilde{u}}(2 \Phi(s) \rho(s))_{s} d s-w(\tilde{u}) \varphi \int_{u}^{\tilde{u}}(2 \Phi(s) \rho(s))_{s} d s \\
& +w(u) \tilde{\varphi} \Phi(u)\left(|\nabla u|^{2}+\varphi^{2}\right)+w(\tilde{u}) \varphi \Phi(\tilde{u})\left(|\nabla \tilde{u}|^{2}+\tilde{\varphi}^{2}\right) \\
& -w(u) \varphi \Phi(u)(\nabla u \cdot \nabla \tilde{u}+\varphi \tilde{\varphi})-w(\tilde{u}) \tilde{\varphi} \Phi(\tilde{u})(\nabla u \cdot \nabla \tilde{u}+\varphi \tilde{\varphi}) \\
= & w(u) \tilde{\varphi} \int_{u}^{\tilde{u}}(2 \Phi(s) \rho(s))_{s} d s-w(\tilde{u}) \varphi \int_{u}^{\tilde{u}}(2 \Phi(s) \rho(s))_{s} d s \\
& +\tilde{\varphi} \nabla G(u) \cdot \nabla u+\varphi \nabla G(\tilde{u}) \cdot \nabla \tilde{u}-\varphi \nabla G(u) \cdot \nabla \tilde{u}-\tilde{\varphi} \nabla G(\tilde{u}) \cdot \nabla u,
\end{aligned}
$$

where we define $G(s)=\int w(s) \Phi(s) d s$. Integrating by parts, we have

$$
\begin{aligned}
& \int_{\Sigma}[\tilde{\varphi} \nabla G(u) \cdot \nabla u+\varphi \nabla G(\tilde{u}) \cdot \nabla \tilde{u}-\varphi \nabla G(u) \cdot \nabla \tilde{u}-\tilde{\varphi} \nabla G(\tilde{u}) \cdot \nabla u] \\
= & \int_{\Sigma}[G(u) \nabla(\varphi \nabla \tilde{u})-G(u) \nabla(\tilde{\varphi} \nabla u)+G(\tilde{u}) \nabla(\tilde{\varphi} \nabla u)-G(\tilde{u}) \nabla(\varphi \nabla \tilde{u})] \\
= & \int_{\Sigma}(G(\tilde{u})-G(u))(\nabla \tilde{\varphi} \cdot \nabla u-\nabla \varphi \cdot \nabla \tilde{u}+\tilde{\varphi} \Delta u-\varphi \Delta \tilde{u}) \\
= & \int_{\Sigma}\left[\sum_{i, j}\left(\tilde{h}_{i j} \tilde{u}_{i} u_{j}-h_{i j} u_{i} \tilde{u}_{j}\right)+\tilde{\varphi}(n f(u)-H \varphi)-\varphi(n f(\tilde{u})-\tilde{H} \tilde{\varphi})\right]\left(\int_{u}^{\tilde{u}} w(s) \Phi(s) d s\right) \\
= & \int_{\Sigma} \sum_{i, j} v_{i j} u_{i} \tilde{u}_{j}\left(\int_{u}^{\tilde{u}} w(s) \Phi(s) d s\right)+\int_{\Sigma}(n f(u) \tilde{\varphi}-n f(\tilde{u}) \varphi) \int_{u}^{\tilde{u}} w(s) \Phi(s) d s,
\end{aligned}
$$

where we have used (2.1), (2.3) and (4.1).

Now, combing (4.9), (4.10) and (4.8), we have

$$
\begin{aligned}
0= & \frac{1}{2} \int_{\Sigma}(w(u) \tilde{\varphi}+w(\tilde{u}) \varphi) \sum_{i, j} v_{i j}^{2} \\
& +\int_{\Sigma} \sum_{i, j} u_{i} \tilde{u}_{j} v_{i j} \int_{u}^{\tilde{u}}\left(w_{s s}(s)+(n-1) w(s) \Phi(s)\right) d s \\
& +\int_{\Sigma}(w(u) \tilde{\varphi}-w(\tilde{u}) \varphi) \int_{u}^{\tilde{u}}\left[(n-1) n(\Phi(s) \rho(s))_{s}-\frac{(n-1) n}{2} f_{s s}(s)\right] d s \\
& +(n-1) n \int_{\Sigma}(f(u) \tilde{\varphi}-f(\tilde{u}) \varphi) \int_{u}^{\tilde{u}} w(s) \Phi(s) d s .
\end{aligned}
$$


Since $(\rho \Phi)_{u}=\rho \Phi_{u}+\rho_{u} \Phi=\rho \Phi_{u}+f \Phi$, by (2.12) we have

$$
2(\rho \Phi)_{u}-f_{u u}=2 \rho \Phi_{u}+2 f \Phi-f_{u u}=-2 f \Phi .
$$

Inserting (4.12) into (4.11), we thus obtain

$$
\begin{aligned}
0= & \frac{1}{2} \int_{\Sigma}(w(u) \tilde{\varphi}+w(\tilde{u}) \varphi) \sum_{i, j} v_{i j}^{2} \\
& +\int_{\Sigma} \sum_{i, j} u_{i} \tilde{u}_{j} v_{i j} \int_{u}^{\tilde{u}}\left(w_{s s}(s)+(n-1) \Phi(s) w(s)\right) d s \\
& +(n-1) n \int_{\Sigma} \tilde{\varphi}\left[-w(u) \int_{u}^{\tilde{u}} f(s) \Phi(s) d s+f(u) \int_{u}^{\tilde{u}} w(s) \Phi(s) d s\right] \\
& +(n-1) n \int_{\Sigma} \varphi\left[w(\tilde{u}) \int_{u}^{\tilde{u}} f(s) \Phi(s) d s-f(\tilde{u}) \int_{u}^{\tilde{u}} w(s) \Phi(s) d s\right] .
\end{aligned}
$$

Now suppose $\left(N, d s^{2}\right)$ satisfies (1.7). Let $\left(r_{0}, r_{1}\right) \subset I$ be an interval so that the coordinate $r$ varies between $r_{0}$ and $r_{1}$ for all points on $M$ and $\tilde{M}$. Similar to Section 3. we let $w$ be a solution to the ODE

$$
w_{u u}+(n-1) \Phi w=0 .
$$

With such a choice of $w$, the second integral on the right side of (4.13) vanishes. To handle the last two integrals, we note that

$$
\begin{aligned}
& f(u) \int_{u}^{\tilde{u}} w(s) \Phi(s) d s-w(u) \int_{u}^{\tilde{u}} f(s) \Phi(s) d s \\
= & \int_{u}^{\tilde{u}} f(u) f(s)\left[\frac{w(s)}{f(s)}-\frac{w(u)}{f(u)}\right] \Phi(s) d s,
\end{aligned}
$$

and

$$
\begin{aligned}
& f(\tilde{u}) \int_{u}^{\tilde{u}} w(s) \Phi(s) d s-w(\tilde{u}) \int_{u}^{\tilde{u}} f(s) \Phi(s) d s \\
= & \int_{u}^{\tilde{u}} f(\tilde{u}) f(s)\left[\frac{w(s)}{f(s)}-\frac{w(\tilde{u})}{f(\tilde{u})}\right] \Phi(s) d s .
\end{aligned}
$$

Thus, we want $w / f$ to have a suitable monotonic property depending on the sign of $\Phi$. If $\Phi \geq 0$, we specify the initial conditions of $w$ at $r=r_{0}$ so that $w\left(r_{0}\right)>0$, $w_{u}\left(r_{0}\right)>0$ and

$$
w_{u}\left(r_{0}\right) f\left(r_{0}\right)-w\left(r_{0}\right) f_{u}\left(r_{0}\right) \geq 0 .
$$

Then, by the argument in Section 3, we have $w>0$ and $w_{u} f-w f_{u} \geq 0$. The latter then implies $w / f$ is monotonically non-decreasing. Hence, it follows from (4.13), (4.14) and (4.15) that $v_{i j}=0$, provided $\varphi>0$ and $\tilde{\varphi}>0$. If $\Phi>0$, by choosing the inequality in (4.16) to be strict, we then have $w_{u} f-w f_{u}>0$. In this case, 
$w / f$ is strictly increasing, and we conclude from (4.13), (4.14) and (4.15) that $u=\tilde{u}$ everywhere on $\Sigma$. As a result, $M$ and $\tilde{M}$ differ by a rotation of $\left(N, d s^{2}\right)$.

The case $\Phi \leq 0$ (and $\Phi<0$ ) are proved in a similar way by choosing the initial conditions of $w$ at $r=r_{1}$ so that $w\left(r_{1}\right)>0, w_{u}\left(r_{1}\right)<0$ and

$$
w_{u}\left(r_{1}\right) f\left(r_{1}\right)-w\left(r_{1}\right) f_{u}\left(r_{1}\right) \leq 0 .
$$

When $\Phi<0$, we choose the inequality in (4.17) to be strict.

Therefore, the discussion above has proved the following theorem, which is a restatement of Theorem 1.1.

Theorem 4.1. Suppose $\left(N, d s^{2}\right)=\left(I \times \mathbb{S}^{n}, \frac{1}{f^{2}(r)} d r^{2}+r^{2} d \sigma\right)$ satisfies (1.7), with either $\Phi \geq 0$ or $\Phi \leq 0$. Let $g$ be a Riemannian metric on $\Sigma=\mathbb{S}^{n}$. Suppose $(\Sigma, g)$ can be isometrically immersed into $\left(N, d s^{2}\right)$ as two hypersurfaces $M$ and $\tilde{M}$, both of which have positive support function. If $M$ and $\tilde{M}$ have the same mean curvature, then $M$ and $\tilde{M}$ have the same second fundamental form. If in addition $\Phi>0$ or $\Phi<0$, then $M$ and $\tilde{M}$ only differ by a rotation of the ambient space $\left(N, d s^{2}\right)$.

Remark 4.1. In view of the above proof, to draw the conclusion $u=\tilde{u}$, it suffices to require the set $\{\Phi>0\}$ is dense in $I$. On the other hand, if the set $\{\Phi=0\}$ contains some open interval $\tilde{I}$, then it is easily checked $\left(N, d s^{2}\right)$ contains a ring $R=\tilde{I} \times \mathbb{S}^{n}$ which is part of a space form. In this case, if $M$ is contained in $R$, we can "translate" $M$ in $R$ by an isometry of that space form. Obviously, such a "translation" preserves the second fundamental form of $M$, but it may not be the restriction of a global isometry of $\left(N, d s^{2}\right)$.

\section{Other RELATED RIGIDities}

In this last section, we will describe several related rigidity results.

First, we consider rigidity of isometric hypersurfaces with the same $\sigma_{2}$-curvature. Recall that the $\sigma_{2}$-curvature of a hypersurface $M$ is defined by

$$
\sigma_{2}(h)=\sum_{i<j} \kappa_{i} \kappa_{j}
$$

where $\left\{\kappa_{i}\right\}$ are the principal curvature of $M$.

Theorem 5.1. Let $\left(N, d s^{2}\right)=\left(I \times \mathbb{S}^{n}, \frac{1}{f^{2}(r)} d r^{2}+r^{2} d \sigma\right)$ be a warped product space with $\Phi \neq 0$. Let $g$ be a Riemannian metric on $\Sigma=\mathbb{S}^{n}$. If $(\Sigma, g)$ can be isometrically immersed into $\left(N, d s^{2}\right)$ as a hypersurface $M$ with nowhere vanishing mean curvature and nowhere vanishing support function, then the infinitesimal rigidity of $M$ with fixed $\sigma_{2}$-curvature holds. Precisely, this means that if $(\Sigma, g)$ admits a family of isometric immersion $\left\{\iota_{t}\right\}$ into $\left(N, d s^{2}\right)$ such that $M_{t}=\iota_{t}(\Sigma)$ has the same $\sigma_{2}$-curvature and $M_{0}=M$, then the linearization of the second fundamental form of $M_{t}$ at $M$ is trivial. 
Proof. We use the same notations in Section 3. By the assumptions,

$$
\dot{\sigma}_{2}(h)=\left.\frac{d \sigma_{2}(h)}{d t}\right|_{t=0}=0 ; \dot{g}_{i j}=\left.\frac{d g_{i j}}{d t}\right|_{t=0}=0 .
$$

Hence, the linearization of (2.5) gives

$$
\frac{n}{2} f_{u u} \dot{u}-(n-2)\left(f \Phi+\rho \Phi_{u}\right) \dot{u}-2 \varphi \dot{\varphi} \Phi-\varphi^{2} \Phi_{u} \dot{u}=0 .
$$

Multiplying $\dot{u}$ in both sides of (5.3) and integrating on $(\Sigma, g)$, we have

$$
\begin{aligned}
0= & \frac{n}{2} \int_{\Sigma} f_{u u} \dot{u}^{2}-2 \int_{\Sigma}(f \dot{u}-\nabla u \cdot \nabla \dot{u}) \Phi \dot{u}-\int_{\Sigma}\left(2 \rho-|\nabla u|^{2}\right) \Phi_{u} \dot{u}^{2} \\
& -(n-2) \int_{\Sigma}\left(f \Phi+\rho \Phi_{u}\right) \dot{u}^{2} \\
= & \frac{n}{2} \int_{\Sigma}\left(f_{u u}-2 f \Phi-2 \rho \Phi_{u}\right) \dot{u}^{2}+\int_{\Sigma} \Phi \nabla u \cdot \nabla \dot{u}^{2}+\int_{\Sigma}|\nabla u|^{2} \Phi_{u} \dot{u}^{2} \\
= & \frac{n}{2} \int_{\Sigma}\left(f_{u u}-2 f \Phi-2 \rho \Phi_{u}\right) \dot{u}^{2}-\int_{\Sigma} \Phi(\Delta u) \dot{u}^{2} \\
= & \frac{n}{2} \int_{\Sigma}\left(f_{u u}-2 f \Phi-2 \rho \Phi_{u}\right) \dot{u}^{2}+\int_{\Sigma} \Phi(H \varphi-n f) \dot{u}^{2} \\
= & \frac{n}{2} \int_{\Sigma}\left(f_{u u}-4 f \Phi-2 \rho \Phi_{u}\right) \dot{u}^{2}+\int_{\Sigma} \Phi H \varphi \dot{u}^{2} .
\end{aligned}
$$

Thus, by (2.12), we have $\dot{u}=0$ since $\Phi H \varphi \neq 0$. In view of (3.2) and (3.3), we conclude $\dot{h}_{i j}=0$.

Remark 5.1. Unlike the fixed mean curvature problem, Theorem 5.1 does not need the assumption (1.7), and the Codazzi equations are not used in the proof.

By imposing a stronger assumption on $\Phi$, we can prove the global rigidity result stated in Theorem 1.2.

Theorem 5.2. Let $\left(N, d s^{2}\right)=\left(I \times \mathbb{S}^{n}, \frac{1}{f^{2}(r)} d r^{2}+r^{2} d \sigma\right)$ be a warped product space with $\Phi \Phi_{u}>0$. Let $g$ be a Riemannian metric on $\Sigma=\mathbb{S}^{n}$. Suppose $(\Sigma, g)$ can be isometrically immersed into $\left(N, d s^{2}\right)$ as two hypersurfaces $M$ and $\tilde{M}$. If $M$ and $\tilde{M}$ have the same $\sigma_{2}$-curvature, then they differ by a rotation of the ambient space $\left(N, d s^{2}\right)$.

Proof. We make use of the maximum principle. To illustrate the idea, we first give another proof of the infinitesimal rigidity in this setting. Using (2.1), (2.12) and (3.2), we can rewrite (5.3) as

$$
n f \Phi \dot{u}+2 \nabla u \cdot \nabla \dot{u} \Phi+|\nabla u|^{2} \Phi_{u} \dot{u}=0 .
$$

At the maximum and minimum points of the function $\dot{u}$, we have $\nabla \dot{u}=0$, which implies $\dot{u}=0$ at these points by the assumption $\Phi \Phi_{u}>0$. Thus, we have $\dot{u}=0$ everywhere on $\Sigma$. 
To prove the global rigidity, we use the same notations from Section 4 . By (4.2), we have

$$
\frac{n}{2} f_{u}-(n-2) \rho \Phi-\varphi^{2} \Phi=\frac{n}{2} f_{u}(\tilde{u})-(n-2) \tilde{\rho} \Phi(\tilde{u})-\tilde{\varphi}^{2} \Phi(\tilde{u}) .
$$

We can rewrite it as

$$
\begin{aligned}
& \frac{n}{2} \int_{u}^{\tilde{u}} f_{s s}(s) d s \\
= & n \int_{u}^{\tilde{u}}(\rho \Phi)_{s} d s+|\nabla u|^{2} \Phi(u)-|\nabla \tilde{u}|^{2} \Phi(\tilde{u}) \\
= & n \int_{u}^{\tilde{u}}\left(f \Phi+\rho \Phi_{s}\right) d s+\nabla(u-\tilde{u}) \cdot \nabla(u+\tilde{u}) \Phi(u)+|\nabla \tilde{u}|^{2}(\Phi(u)-\Phi(\tilde{u})),
\end{aligned}
$$

which together with (2.12) implies

$$
n \int_{u}^{\tilde{u}} f \Phi d s=\nabla(u-\tilde{u}) \cdot \nabla(u+\tilde{u}) \Phi(u)-|\nabla \tilde{u}|^{2} \int_{u}^{\tilde{u}} \Phi_{s} d s .
$$

At the maximum and minimum points of the function $u-\tilde{u}$, we have $\nabla(u-\tilde{u})=0$. Thus, (5.7) implies $u=\tilde{u}$ at these points since $\Phi \Phi_{u}>0$. Therefore, $u=\tilde{u}$ on the entire $\Sigma$, which implies $M$ and $\tilde{M}$ differ by a rotation of $\left(N, d s^{2}\right)$.

The techniques used in Sections 3 and 4 indeed relate to a revisit of the rigidity of isometric embeddings of surfaces into space forms. We give a brief review of the history of this rigidity problem. It is known that the infinitesimal rigidity of closed convex surfaces in Euclidean spaces was shown by Cohn-Vossen [15] and was simplified by Blaschke [3] using Minkowski identities. The infinitesimal rigidity in hyperbolic spaces was discussed by Lin-Wang [30]. In [24], the first and the third authors gave an alternative proof of the infinitesimal rigidity for convex surfaces in space forms. The global rigidity was obtained by Cohn-Vossen [14] for convex surfaces in Euclidean spaces. In space forms, these rigidities are also known valid (cf. [17, 21]).

Below we give an alternate proof of the global rigidity using the methods from Sections 3 and 4 .

Theorem 5.3. Suppose $\left(N, d s^{2}\right)$ is a 3-dimensional space form. Let $g$ be a metric on the 2-sphere $\mathbb{S}^{2}$. If $\left(\mathbb{S}^{2}, g\right)$ can be isometrically embedded into $\left(N, d s^{2}\right)$ as a strictly convex surface $\Sigma$, then the infinitesimal rigidity and global rigidity of $\Sigma$ hold.

Proof. In a space form with constant sectional curvature $-k$, we have

$$
\Phi=0, f_{u}=k .
$$

Thus, the Gauss equation becomes

$$
\frac{\operatorname{det} h}{\operatorname{det} g}=K+k \text {. }
$$

By "translating" $\Sigma$ in $\left(N, d s^{2}\right)$, we can always assume that the support function $\varphi$ of $\Sigma$ is positive since $\Sigma$ is strictly convex. 
First we consider the infinitesimal rigidity. The linearization of the Gauss-Codazzi equations gives

$$
\begin{gathered}
h_{11} \dot{h}_{22}+h_{22} \dot{h}_{11}-2 h_{12} \dot{h}_{12}=0, \\
\dot{h}_{i j, k}=\dot{h}_{i k, j} .
\end{gathered}
$$

By (3.3), we have

$$
\dot{u}_{i j}=k \dot{u} \delta_{i j}-\dot{h}_{i j} \varphi-h_{i j} \dot{\varphi} .
$$

Integrating by parts, we have

$$
0=\int_{\Sigma}\left[\left(f(u) \dot{u}_{1}\right)_{1} \dot{h}_{22}+\left(f(u) \dot{u}_{2}\right)_{2} \dot{h}_{11}-\left(f(u) \dot{u}_{1}\right)_{2} \dot{h}_{21}-\left(f(u) \dot{u}_{2}\right)_{1} \dot{h}_{12}\right]
$$

On the other hand, we have

$$
\left(f(u) \dot{u}_{i}\right)_{j}=f_{u} u_{j} \dot{u}_{i}+f(u) \dot{u}_{i j}=k u_{j} \dot{u}_{i}+f(u)\left(k \dot{u} \delta_{i j}-\dot{h}_{i j} \varphi-h_{i j} \dot{\varphi}\right) .
$$

Inserting the above equality into (5.10), we have

$$
\begin{aligned}
& \int_{\Sigma}\left[k u_{1} \dot{u}_{1} \dot{h}_{22}+k f(u) \dot{u} \dot{h}_{22}+k u_{2} \dot{u}_{2} \dot{h}_{11}+k f(u) \dot{u} \dot{h}_{11}-k u_{1} \dot{u}_{2} \dot{h}_{12}-k u_{2} \dot{u}_{1} \dot{h}_{21}\right] \\
& -2 \int_{\Sigma}\left(\dot{h}_{11} \dot{h}_{22}-\dot{h}_{12} \dot{h}_{21}\right) \varphi=0 .
\end{aligned}
$$

Here we have used (5.8). Integrating by part, we also have

$$
\begin{aligned}
& \int_{\Sigma}\left[k u_{1} \dot{u}_{1} \dot{h}_{22}+k u_{2} \dot{u}_{2} \dot{h}_{11}-k u_{1} \dot{u}_{2} \dot{h}_{12}-k u_{2} \dot{u}_{1} \dot{h}_{21}\right] \\
= & -k \int_{\Sigma} \dot{u}\left[\left(u_{1} \dot{h}_{22}\right)_{1}+\left(u_{2} \dot{h}_{11}\right)_{2}-\left(u_{2} \dot{h}_{12}\right)_{1}-\left(u_{1} \dot{h}_{21}\right)_{2}\right] \\
= & -k \int_{\Sigma} \dot{u}\left[u_{1}\left(\dot{h}_{221}-\dot{h}_{122}\right)+u_{2}\left(\dot{h}_{112}-\dot{h}_{211}\right)+u_{11} \dot{h}_{22}+u_{22} \dot{h}_{11}-2 u_{12} \dot{h}_{12}\right] \\
= & -k \int_{\Sigma} \dot{u}\left[\dot{h}_{22}\left(f(u)-h_{11} \varphi\right)+\dot{h}_{11}\left(f(u)-h_{22} \varphi\right)-2 \dot{h}_{12}\left(-h_{12} \varphi\right)\right] \\
= & -k \int_{\Sigma} \dot{u}\left(\dot{h}_{11}+\dot{h}_{22}\right) f(u) .
\end{aligned}
$$

Combing (5.11) and (5.12), we obtain

$$
\int_{\Sigma}\left(\dot{h}_{11} \dot{h}_{22}-\dot{h}_{12}^{2}\right) \varphi=0 .
$$

At any given point, by computing in a normal coordinate chart that diagonalizes $h_{i j}$, it is easily seen that (5.8), together with the convexity of $\Sigma$, implies

$$
\dot{h}_{11} \dot{h}_{22}-\dot{h}_{12}^{2} \leq 0 \text {. }
$$

Hence, from (5.13), we obtain $\dot{h}=0$. 
Next, we modify the previous argument to prove the global rigidity. Suppose $\Sigma$ and $\tilde{\Sigma}$ are two isometrically embedded convex surfaces. Suppose their second fundamental forms are $h$ and $\tilde{h}$, respectively. Then, $v=\tilde{h}-h$ satisfies

$$
\begin{aligned}
& h_{11} v_{22}+h_{22} v_{11}-2 h_{12} v_{12}+v_{11} v_{22}-v_{12}^{2}=0, \\
& \tilde{h}_{11} v_{22}+\tilde{h}_{22} v_{11}-2 \tilde{h}_{12} v_{12}-v_{11} v_{22}+v_{12}^{2}=0,
\end{aligned}
$$

by the Gauss equations. Thus, we have

$$
\left(h_{11}+\tilde{h}_{11}\right) v_{22}+\left(h_{22}+\tilde{h}_{22}\right) v_{11}-2\left(h_{12}+\tilde{h}_{12}\right) v_{12}=0 .
$$

If we compute in a normal coordinate chart that diagonalizes $h+\tilde{h}$, then (5.14) and the convexity of $\Sigma$ and $\tilde{\Sigma}$ show $v_{11}$ and $v_{22}$ have different sign. Thus, we have

$$
v_{11} v_{22}-v_{12}^{2} \leq 0 \text {. }
$$

The Codazzi equations imply

$$
v_{i j, k}=v_{i k, j}
$$

Integrating by part, we have

$$
\begin{aligned}
0= & \int_{\Sigma}\left[\left(f(\tilde{u}) u_{1}-f(u) \tilde{u}_{1}\right)_{1} v_{22}+\left(f(\tilde{u}) u_{2}-f(u) \tilde{u}_{2}\right)_{2} v_{11}\right. \\
& \left.-\left(f(\tilde{u}) u_{1}-f(u) \tilde{u}_{1}\right)_{2} v_{21}-\left(f(\tilde{u}) u_{2}-f(u) \tilde{u}_{2}\right)_{1} v_{12}\right] .
\end{aligned}
$$

On the other hand, we have

$$
\begin{aligned}
& \left(f(\tilde{u}) u_{i}-f(u) \tilde{u}_{i}\right)_{j} \\
= & f(\tilde{u}) u_{i j}-f(u) \tilde{u}_{i j}+k\left(\tilde{u}_{j} u_{i}-u_{j} \tilde{u}_{i}\right) \\
= & f(\tilde{u})\left(f(u) \delta_{i j}-h_{i j} \varphi\right)-f(u)\left(f(\tilde{u}) \delta_{i j}-\tilde{h}_{i j} \tilde{\varphi}\right)+k\left(\tilde{u}_{j} u_{i}-u_{j} \tilde{u}_{i}\right) \\
= & -f(\tilde{u}) h_{i j} \varphi+f(u) \tilde{h}_{i j} \tilde{\varphi}+k\left(\tilde{u}_{j} u_{i}-u_{j} \tilde{u}_{i}\right) .
\end{aligned}
$$

Inserting the above equality into (5.17), we obtain

$$
\begin{aligned}
0 & =\int_{\Sigma}\left[-f(\tilde{u})\left(h_{11} v_{22}+h_{22} v_{11}-2 h_{12} v_{12}\right) \varphi+f(u)\left(\tilde{h}_{11} v_{22}+\tilde{h}_{22} v_{11}-2 \tilde{h}_{21} v_{12}\right) \tilde{\varphi}\right] \\
& =\int_{\Sigma}(f(\tilde{u}) \varphi+f(u) \tilde{\varphi})\left(v_{11} v_{22}-v_{12}^{2}\right) .
\end{aligned}
$$

Here we have used (5.14). Combing (5.15) and (5.18), we conclude $v=0$. Thus, $\Sigma$ and $\tilde{\Sigma}$ have the same first and second fundamental forms. Since $\left(N, d s^{2}\right)$ is a space form, we conclude that $\Sigma$ and $\tilde{\Sigma}$ are same up to an isometry of $\left(N, d s^{2}\right)$.

Acknowledgement. The main part of this work was completed when the first author was visiting Fudan University in 2017. He would like to thank Fudan University for the hospitality and support. The authors also thank Siyuan Lu for helpful conversations. 


\section{REFERENCES}

[1] A.D. Alexandrov, Uniqueness theorems for surfaces in the large I, Vesnik Leningrad Univ. 11, 5-17 (1956).

[2] R. Bartnik, New definition of quasilocal mass, Phys. Rev. Lett., 62 (1989), 2346-2348.

[3] W. Blaschke, Vorlesungen Uber Differentialgeometrie, Vol. 1, Julis Springer, Berlin, 1924.

[4] H. L. Bray, Proof of the Riemannian Penrose inequality using the positive mass theorem. J. Differential Geom. 59 (2001), no. 2, 177-267.

[5] S. Brendle, Constant mean curvature surfaces in warped product space, Publ. Math. Inst. Hautes etudes Sci.(117) 2013, 247-269.

[6] J. D. Brown and J. W. York, Jr., Quasilocal energy in general relativity, in Mathematical aspects of classical field theory (Seattle, WA, 1991), volume 132 of Contemp. Math., pages 129-142. Amer. Math. Soc., Providence, RI, 1992.

[7] J. D. Brown and J. W. York, Jr., Quasilocal energy and conserved charges derived from the gravitational action, Phys. Rev. D (3) 47 (1993), no. 4, 1407-1419.

[8] É. Cartan, Sur les couples de surfaces applicable avec conservation des coupures principles, Bull. Sci. Math. 66 (1942) 1-30.

[9] J. Chang and L. Xiao, The Weyl problem with nonnegative Gauss curvature in hyperbolic space, Canad. J. Math. 67(2015), 107-131.

[10] P.-N. Chen, M.-T. Wang, Y.-K. Wang and S.-T. Yau, Quasi-local energy with respect to a static spacetime, arXiv:1604.02983.

[11] P.-N. Chen and X. Zhang, A rigidity theorem for surfaces in Schwarzschild manifold, arXiv:1802.00887.

[12] S.S. Chern, Deformation of surfaces preserving principal curvature, Differential geometry and complex analysis, H.E. Rauch Menerial Volume (Springer 1985) 155-163.

[13] A. Gervasio Colares and K. Kenmotsu, Isometric deformation of surfaces in $\mathbb{R}^{3}$ preserving the mean curvature function, Pacific J. Math. 136 (1989) 71-80.

[14] E. Cohn-Vossen, Zwei Satze uber die Starrheit der Eiflachen, Nach. Gesell-schaft Wiss, Gottingen, Math. Phys. KL, 1927: 125.134.

[15] E. Cohn-Vossen, Unstarre geochlossene Flachen, Math. Ann., 1930, 102: 10.29.

[16] J. Corvino, Scalar curvature deformation and a gluing construction for the Einstein constraint equations, Comm. Math. Phys. 214 (2000), 137-189.

[17] M. Dajczer, Submanifolds and Isometric immersions, Mathematical Lecture Series 13, 1990.

[18] P. Guan, J. Li and M.-T. Wang, A volume preserving flow and the isoperimetric problem in warped product spaces, arXiv:1609.08238.

[19] P. Guan and S. Lu, Curvature estimates for immersed hypersurfaces in Riemannian manifolds, Invent. math. 208 (2017), no. 1, 191-215.

[20] P. Guan, Z. Wang, and X. Zhang A proof of the Alexanderov's uniqueness theorem for convex surface in $\mathbb{R}^{3}$, Ann. Inst. H. Poincare Anal. Non Lineaire, (2016) 329-336.

[21] P. Guan and X. Shen, A rigidity theorem for hypersurfaces in higher dimensional space forms, Contemporary Mathematics, AMS. V.644, 2015. pp. 61-65.

[22] G. Huisken and T. Ilmanen, The inverse mean curvature flow and the Riemannian Penrose inequality, J. Differential Geom. 59 (2001), no. 3, 353-437.

[23] K. Kenmotsu, An intrinsic characterization of $H$-deformable surfaces, J. London Math. Soc. 49 (1994) 555-568.

[24] C. Li and Z. Wang, The Weyl problem in warped product spaces, arXiv:1603.01350.

[25] S. Lu, On Weyl's embedding problem in Riemannian manifolds, arXiv:1608.07539.

[26] S. Lu and P. Miao, Minimal hypersurfaces and boundary behavior of compact manifolds with nonnegative scalar curvature, arXiv: 1703.08164 .

[27] H. B. Lawson Jr., Complete minimal surfaces in $\mathbb{S}^{3}$, Ann. of Math. 92 (1970) 335-374. 
[28] H. B. Lawson Jr. and R. de Azevedo Tribuzy, On the mean curvature function for compact surfaces, J. Diff. Geo. (1981) 179-183.

[29] H. Lewy, On the existence of a closed convex sucface realizing a given Riemannian metric, Proceedings of the National Academy of Sciences, U.S.A., Volume 24, No. 2, (1938), 104-106.

[30] C.-Y. Lin and Y.-K. Wang, On isometric embeddings into anti-de sitter spacetimes, Int. Math. Res. Notices, No. 16 (2015), 7130-7161.

[31] S. Montiel, Unicity of constant mean curvature hypersurfaces in some Riemannian manifolds, Indiana Univ. Math. J. 48, 711-748 (1999)

[32] L. Nirenberg, The Weyl and Minkowski problem in differential geometry in the large, Commun. Pure Appl. Math. 6 (1953), no. 3, 337-394.

[33] A. V. Pogorelov, Regularity of a convex surface with given Gaussian curvature, (Russian) Mat. Sbornik N.S. 31(73), (1952), no. 1, 88-103.

[34] A.V. Pogorelov, Some results on surface theory in the large, Adv. Math. 1 1964, fasc. 2, 191-264.

[35] A.V. Pogorelov, Extrinsic Geometry of Convex Surfaces, Translations of mathematical mongraphs, Vol. 35, AMS, 1973.

[36] W. Scherrer, Die Grundgleichungen der Flächentheorie II, Comm. Math. Helv., 32 (1957), 7384.

[37] A. Svec, Determination of a surface by its mean curvature, Casopis pro pestovani matematiky, 103 (1978), 175-180.

[38] R. Tribuzy, A characterization of tori with constant mean curvature in a space form, Bol. Soc. Brasil. Mat. 11 (1980) 259-274.

[39] H. Weyl, Uber die Bestimmung einer geschlossenen konvexen Flache durch ihr Linienelement, Vierteljahrsschrift der naturforschenden Gesellschaft, Zurich, 61, (1916), 40-72.

[40] M.-T. Wang and S.-T. Yau, Quasilocal mass in general relativity, Phys. Rev. Lett. 102 (2009), no. 2, no. 021101,4 pp.

[41] M.-T. Wang and S.-T. Yau, Isometric embeddings into the Minkowski space and new quasi-local mass, Comm. Math. Phys. 288 (2009), no. 3, 919-942.

[42] R. Schoen and S.-T. Yau, On the proof of the positive mass conjecture in general relativity, Commun. Math. Phys. 65 (1979), no. 1, 45-76.

[43] M. Umehara, A characterization of compact surfaces with constant mean curvature, Proc. Amer. Math. Soc., 108 (1990) 483-489.

(Chunhe Li) School of Mathematical Sciences, University of Electronic Science and Technology of China, Chengdu, China

E-mail address: chli@fudan.edu.cn

(Pengzi Miao) Department of Mathematics, University of Miami, Coral Gables, FL 33146, USA.

E-mail address: pengzim@math.miami.edu

(Zhizhang Wang) School of Mathematical Sciences, Fudan University, Shanghai, CHINA

E-mail address: zzwang@fudan.edu.cn 